\title{
Interações medicamentosas potenciais em idosos com síndrome metabólica
}

\author{
Potential drug interactions in the elderly with metabolic syndrome \\ Posibles interacciones farmacológicas en ancianos con síndrome metabólico
}

Recebido: 09/07/2021 | Revisado: 17/07/2021 | Aceito: 18/07/2021 | Publicado: 26/07/2021

\author{
Tuany Santos Souza \\ ORCID: https://orcid.org/0000-0003-0165-4201 \\ Universidade Estadual do Sudoeste da Bahia, Brasil \\ E-mail: tuanysouza.s@uesb.edu.br \\ Ana Flávia Souto Figueiredo Nepomuceno \\ ORCID: https://orcid.org/0000-0002-3489-0959 \\ Universidade Estadual do Sudoeste da Bahia, Brasil \\ E-mail: anaflaviafigueiredo@outlook.com \\ Silvania Moraes Costa \\ ORCID: https://orcid.org/0000-0002-6607-6177 \\ Universidade Estadual do Sudoeste da Bahia, Brasil \\ E-mail: silvaniamoraescosta@ hotmail.com \\ Yndiara Novaes Santos Oliveira \\ ORCID: https://orcid.org/0000-0001-7222-938X \\ Universidade Estadual do Sudoeste da Bahia, Brasil \\ E-mail: yndiara@msn.com \\ José Ailton de Oliveira Carneiro \\ ORCID: https://orcid.org/0000-0001-5095-0301 \\ Universidade Estadual do Sudoeste da Bahia, Brasil \\ E-mail: hitoef@yahoo.com.br \\ Cézar Augusto Casotti \\ ORCID: https://orcid.org/0000-0001-6636-8009 \\ Universidade Estadual do Sudoeste da Bahia, Brasil \\ E-mail: cacasotti@uesb.edu.br
}

\section{Resumo}

A síndrome Metabólica (SM) é um conjunto de manifestações clínicas crônicas e complexas que possui alta prevalência em idosos, levando-os a utilizar múltiplos medicamentos e tornando-os mais suscetíveis ao aparecimento de interações medicamentosas potenciais (IMP). Objetivou-se estimar a prevalência e fatores associados a interações medicamentosas potenciais em idosos diagnosticados com síndrome metabólica. Trata-se de um estudo transversal, de base populacional e domiciliar realizado com 118 idosos com diagnóstico de SM confirmado, segundo critérios do National Cholesterol Education Program's - Adult Treatment Panel III; as IMP foram identificadas utilizando o programa Medscape ${ }^{\circledR}$. A análise descritiva e bivariada dos dados foi realizada no software SPSS v.21. Avaliou-se a associação entre as variáveis por meio do teste qui-quadrado, adotando-se o nível de significância $\mathrm{p}<0,05$. A prevalência de IMP foi de $27,1 \%$ e esteve significativamente associada a utilização de serviços de saúde ( $\mathrm{p}=0,04)$, com os critérios de diagnóstico para SM ( $\mathrm{p}=0,05)$ e com o uso de tabaco ( $\mathrm{p}=0,03)$. Obteve-se um total de 145 IMP, com média de 4,5 por paciente. Quanto à classificação da gravidade, 76,6\% das IMP apresentavam necessidade de monitoramento; $13,2 \%$ foram classificadas como menor e 10,2\% como graves. Conclui-se que os idosos com SM apresentaram uma prevalência de IMP considerável, estando associada a utilização de serviços de saúde, aos critérios de diagnósticos da SM e ao uso de tabaco. Sugere-se a necessidade de acompanhamento desses idosos por parte da equipe de saúde, principalmente pelo farmacêutico.

Palavras chave: Interações medicamentosas; Idosos; Síndrome X Metabólica; Saúde pública.

\begin{abstract}
Metabolic syndrome (MS) is a set of chronic and complex clinical manifestations that has a high prevalence in the elderly, leading them to use multiple drugs and making them more susceptible to the appearance of potential drug interactions (PMI). This study aimed to estimate the prevalence and factors associated with potential drug interactions in elderly people diagnosed with metabolic syndrome. This is a cross-sectional, population-based and household study carried out with 118 elderly people with a confirmed diagnosis of MS, according to criteria of the National Cholesterol Education Program's - Adult Treatment Panel III; IMPs were identified using the Medscape ${ }^{\circledR}$ program. Descriptive and bivariate data analysis was performed using SPSS v.21 software. The association between the variables was evaluated using the chi-square test, adopting a significance level of $p<0.05$. The prevalence of PIM was $27.1 \%$ and was significantly associated with the use of health services $(\mathrm{p}=0.04)$, with the diagnostic criteria for MS $(\mathrm{p}=0.05)$ and with tobacco use $(\mathrm{p}=0.03)$. A total of 145 IMPs were obtained, with an average of 4.5 per patient. As for the classification of severity, $76.6 \%$ of the IMPs needed monitoring; $13.2 \%$ were classified as minor and $10.2 \%$ as
\end{abstract}


severe. It is concluded that elderly people with MS had a considerable prevalence of PID, which was associated with the use of health services, the criteria for diagnosing MS and tobacco use. The need for monitoring these elderly people by the health team, especially by the pharmacist, is suggested.

Keywords: Drug interactions; Seniors; Metabolic X Syndrome; Public health.

\section{Resumen}

El síndrome metabólico (SM) es un conjunto de manifestaciones clínicas crónicas y complejas que tiene una alta prevalencia en los ancianos, lo que los lleva a utilizar múltiples fármacos y los hace más susceptibles a la aparición de potenciales interacciones medicamentosas (PMI). Este estudio tuvo como objetivo estimar la prevalencia y los factores asociados con posibles interacciones medicamentosas en personas mayores diagnosticadas con síndrome metabólico. Se trata de un estudio transversal, poblacional y domiciliario realizado con 118 ancianos con diagnóstico confirmado de EM, según criterios del Programa Nacional de Educación en Colesterol - Panel III de Tratamiento de Adultos; Los IMP se identificaron mediante el programa Medscape ${ }^{\circledR}$. El análisis de datos descriptivo y bivariado se realizó con el software SPSS v.21. La asociación entre las variables se evaluó mediante la prueba de chi-cuadrado, adoptando el nivel de significancia de $\mathrm{p}<0.05$. La prevalencia de PIM fue del $27,1 \%$ y se asoció significativamente con el uso de los servicios de salud $(\mathrm{p}=0,04)$, con los criterios diagnósticos de $\mathrm{SM}(\mathrm{p}=0,05)$ y con el tabaquismo ( $\mathrm{p}$ $=0,03$ ). Se obtuvieron un total de $145 \mathrm{IMP}$, con una media de 4,5 por paciente. En cuanto a la clasificación de gravedad, el 76,6\% de los PMI precisaron seguimiento; El 13,2\% se clasificaron como leves y el 10,2\% como graves. Se concluye que las personas mayores con EM tenían una prevalencia considerable de EPI, la cual se asoció con el uso de los servicios de salud, los criterios para el diagnóstico de EM y el tabaquismo. Se sugiere la necesidad de seguimiento de estos ancianos por parte del equipo de salud, especialmente por parte del farmacéutico.

Palabras clave: Interacciones farmacológicas; Ancianos; Síndrome X metabólico. Salud pública.

\section{Introdução}

O aumento acelerado da população idosa tem contribuído ao longo dos anos para uma série de mudanças no perfil epidemiológico, no qual se evidenciam principalmente as doenças crônicas não transmissíveis (DCNT), como as principais causas de morbimortalidade (Barreto, Carreira \& Marcon, 2015; Veras \& Oliveira, 2018). Dentre estas doenças, destaca-se a Síndrome Metabólica (SM) que atinge de 20 a 30\% da população mundial, podendo chegar até 65,3\% no Brasil, e se refere a um conjunto de fatores inter-relacionados, que contribuem diretamente para uma apresentação clínica complexa (Lima et al., 2017).

Segundo a I Diretriz Brasileira de Diagnóstico e Tratamento da Síndrome Metabólica (I-DBSM) (Brasil, 2005), que se baseia nos critérios definidos pelo National Cholesterol Education Program's - Adult Treatment Panel III (NCEP - ATP III, 2001), o diagnóstico de SM é confirmado quando estão presentes, simultaneamente, no mínimo três dos cinco critérios, sendo eles: hipertensão arterial (HAS), intolerância à glicose, aumento de triglicerídeos (TG), baixo nível de colesterol do tipo HDL e aumento da circunferência abdominal. Soma-se a isso, fatores comportamentais como os hábitos alimentares, etilismo e tabagismo e principalmente o sedentarismo, sendo este último pontuado no estudo de Coelho et al. (2021), como um importante ponto de influência para o desenvolvimento de patologias metabólicas. Assim, por se tratar de uma condição clínica multifatorial, a terapia da SM, de modo geral, inclui recursos farmacológicos que levam a prática de polifarmácia (Ramos et al., 2016).

Cabe destacar, no entanto, que a polifarmácia trata-se de um importante fator de impacto na saúde e segurança do paciente idoso, tendo em vista que essa prática contribui para o aparecimento de reações adversas a medicamentos (RAM) e interações medicamentosas potenciais (IMP), que se manifestam quando a resposta farmacológica e/ou a toxicidade de um fármaco é alterada pela presença de outro (Tavares et al., 2018). Neste sentido, Ferreira e Soler (2020), destacam a necessidade de fomentar estratégias voltadas ao cuidado do paciente, através de práticas seguras no contexto do uso de medicamentos, visando contribuir diretamente na melhoraria da assistência.

Assim, tendo em vista a realidade epidemiológica do Brasil no que concerne a alta prevalência de SM em idosos associada à necessidade da polimedicação e a consequente propensão ao aparecimento de eventos adversos, somado a escassez de estudos de base populacional que discutam o conhecimento acerca da ocorrência de IMP em idosos residentes em 
comunidade, existe a necessidade de aumentar as discussões sobre a influência dessas interações na saúde e qualidade de vida dos pacientes idosos portadores deste agravo. Nesta perspectiva, este estudo tem como objetivo estimar a prevalência de interações medicamentosas potenciais e identificar os fatores associados a estas interações em idosos com síndrome metabólica.

\section{Metodologia}

Trata-se de um estudo com delineamento transversal, que analisou dados da pesquisa epidemiológica de base populacional e domiciliar, intitulada "Condições de Saúde e Estilo de Vida de idosos residentes em município de pequeno porte", aprovada pelo Comitê de Ética em Pesquisa da Universidade Estadual do Sudoeste da Bahia (CEP-UESB) sob CAAE: 56017816.2.0000.0055, número do parecer 1.575.825 e atende aos requisitos da Resolução 466/2012 do Conselho Nacional de Saúde (Brasil, 2012).

Este município possui população estimada em 4.767 habitantes, dos quais, cerca de 15,7\% são indivíduos idosos (IBGE, 2010). Inicialmente foi realizado um censo da população idosa, onde foram incluídos no estudo indivíduos com idade igual ou superior a 60 anos, com moradia fixa na zona urbana, não institucionalizados, e com diagnostico de síndrome metabólica, avaliado pelo critério da I-DBSM (Brasil, 2005). Foram excluídos idosos residentes na zona rural, que apresentaram déficit cognitivo (pontuação <13 pontos) mediante avaliação do Mini Exame do Estado Mental - MEEM (Folstein, Folstein \& Mchugh, 1975) que não possuíam acompanhante que os auxiliassem nas respostas e aqueles não encontrados em sua residência após 3 tentativas de visita em dias e horários alternados.

Foram localizados 379 idosos, de ambos os sexos, com idade $\geq 60$ anos cadastrados nas Unidades de Saúde da Família (USF) e residentes na zona urbana do município de Aiquara/BA. Ressalta-se que para este estudo participaram apenas 118 idosos que obtiveram o diagnóstico confirmado para SM. A descrição do processo de inclusão dos idosos no estudo encontra-se na Figura 1.

A coleta dos dados ocorreu de janeiro a julho de 2015, em três etapas: I. Inquérito domiciliar, com aplicação de questionário para avaliação das características sócio-demográficas, condições de saúde e estilo de vida; II. Avaliação clínica, onde foi realizada a aferição da pressão arterial, segundo os procedimentos preconizados pela VII Diretriz Brasileira de Hipertensão Arterial (Malachias et al., 2016) além da mensuração de medidas antropométricas, de acordo com a técnica de Petroski (2011); III. Coleta de amostra sanguínea, feita com agendamento prévio, onde os idosos foram orientados a fazer jejum noturno de 12 horas. As duas últimas etapas foram realizadas na Secretaria Municipal de Saúde, por profissionais capacitados, respeitando-se as normas de biossegurança.

Após a coleta, as amostras de sangue foram encaminhadas para processamento e análises em um Laboratório de Saúde Pública, localizado em um Centro de Referência em Doenças Endêmicas, no município de Jequié-BA, onde foram realizadas as seguintes determinações bioquímicas: glicemia de jejum, colesterol total e suas frações (HDL-c, LDL-c e VLDLc) e triglicérides. 
Figura 1 - Diagrama do processo de inclusão dos idosos no estudo durante as etapas de coleta. Aiquara-BA, Brasil, 2015.

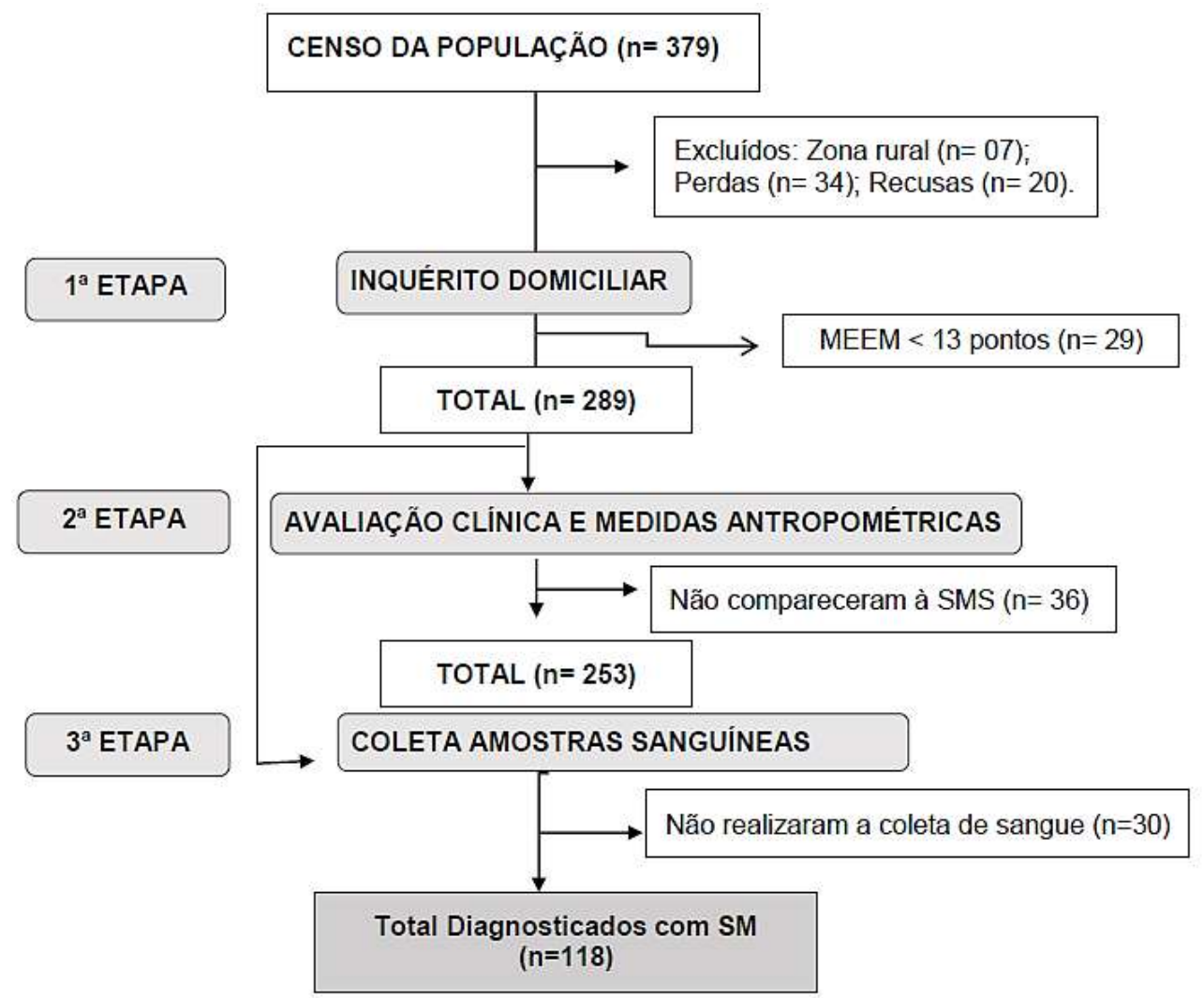

Fonte: Autores.

O diagnóstico de SM foi confirmado de acordo com a I-DBSM (Brasil, 2005), que se baseia nos critérios do NCEPATP III (2001), levando-se em consideração a alteração em pelo menos três, dos seguintes componentes: pressão arterial sistólica $\geq 130$ ou pressão arterial diastólica $\geq 85 \mathrm{mmHg}$ ou uso de medicamento anti-hipertensivo; glicemia de jejum $\geq 110$ $\mathrm{mg} / \mathrm{dL}$ ou uso de hipoglicemiantes; triglicerídeos $\geq 150 \mathrm{mg} / \mathrm{dL}$; colesterol $\mathrm{HDL}<40 \mathrm{mg} / \mathrm{dL}$ para os homens e $<50 \mathrm{mg} / \mathrm{dL}$ para as mulheres e circunferência abdominal $\geq 88 \mathrm{~cm}$ para as mulheres $\geq 102 \mathrm{~cm}$ para os homens.

Como variável dependente deste estudo, elencou-se as potenciais interações medicamentosas (IMP), as quais foram verificadas por meio da consulta à base de dados Medscape Drug Interaction Checke, que classifica as interações quanto ao seu risco como: grave, necessita de monitoramento e leve; e quanto ao mecanismo como: farmacodinâmico, farmacocinético ou desconhecido. As variáveis independentes foram selecionadas e agrupadas em três blocos: sociodemográficas; comportamentais; exames laboratoriais e condições de saúde.

As variáveis sociodemográficas consistiram em: sexo (feminino, masculino); faixa etária em anos (60-69, 70-79 e $\geq$ 80); cor da pele por meio do autorrelato (negro e não-negro); escolaridade em anos de estudo ( $\leq 8$ anos e $>8$ anos); arranjo familiar (com companheiro e sem companheiro) renda ( $\leq 1$ salário mínimo; $>1$ salário mínimo). As variáveis comportamentais corresponderam ao consumo de bebidas alcóolicas ( partir do Questionário Internacional de Atividade Física - IPAQ (suficientemente ativo ou insuficientemente ativo) (Mazo e Benedetti, 2010); busca aos serviços de saúde por ano ( $\geq 2$ vezes/ano; 1 vez/ano; nenhuma).

As variáveis laboratoriais e de condições de saúde incluíram: determinação das concentrações séricas de colesterol total (VR $<200 \mathrm{mg} / \mathrm{dL}$ ); Sobrepeso que foi verificado por meio do incide de massa corpórea (Baixo peso: $<22 \mathrm{~kg} / \mathrm{m}^{2} \mathrm{Eutrófico:}$ $22,1 \mathrm{~kg} / \mathrm{m}^{2}-26,9 \mathrm{~kg} / \mathrm{m}^{2}$ Sobrepeso: $\left.>27 \mathrm{~kg} / \mathrm{m}^{2}\right)($ Lipschitz, 1994) uso de medicamentos (hipolipemiantes, hipoglicemiantes e 
anti-hipertensivos) para algum dos componentes da SM (sim e não) (Brasil, 2005), descritos de acordo com a classificação Anatomical Therapeutic Chemical (ATC), da Organização Mundial de Saúde (OMS, 2009).

A análise estatística foi realizada de forma descritiva através da distribuição de frequências absolutas e relativas para as variáveis categóricas e médias e desvio-padrão para as variáveis contínuas. A prevalência das IMP foi estimada levando-se em consideração a população estudada e o total de idosos expostos. Na análise bivariada a associação entre as IMP e as variáveis independentes foi verificada por meio do teste Qui-quadrado. Em todas as análises o nível de significância adotado foi de 5\% e utilizou-se o programa Statistical Package for Social Sciences para Windows (SPSS v. 21.0, 2013, SPSS, Inc, Chicago, IL).

\section{Resultados}

Participaram do estudo 118 idosos com diagnóstico de SM, cujas informações sociodemográficas, comportamentais e de condições de saúde estão descritas na Tabela 1. Destaca-se que houve predominância de idosos do sexo masculino (72\%), negros (77,8\%), com faixa etária entre 60 a 69 anos (44,9\%), que vivem sem companheiro(a) (59,0\%), com escolaridade menor que oito anos de estudo (96,5\%) e que vivem com baixa renda mensal (87,9\%). Quanto ao nível de atividades físicas, observou-se que a maioria dos idosos são insuficientemente ativos $(55,1 \%)$. Verificou-se que a maioria deles frequentam os serviços de saúde duas ou mais vezes por ano $(85,8 \%)$ e a maioria não consumiam álcool $(85,5 \%)$ e não faziam uso de tabaco $(94,5 \%)$.

Com relação às condições de saúde observou-se que apenas 13,7\% dos idosos apresentaram cinco critérios da SM, sendo que 56,8\% possuíam colesterol total alterado, 50\% LDL-c alterado, a maioria possuía sobrepeso (65\%) e tinham presença de comorbidades $(99,2 \%)$. Além disso, $52,5 \%$ não utilizavam medicamentos para o tratamento da SM. Ademais, observou-se que a prevalência de IMP foi de 27,1\% nos idosos com diagnóstico de SM.

Tabela 1 - Características sociodemográficas, comportamentais e de condições de saúde dos idosos com síndrome metabólica. Aiquara-BA, Brasil, 2015.

\begin{tabular}{lccc}
\hline Variáveis & $\mathbf{N}$ & Frequência & $\%$ \\
\hline Sexo & 118 & & \\
$\quad$ Masculino & & 85 & 72,0 \\
$\quad$ Feminino & 118 & 33 & 28,0 \\
Faixa etária & & 53 & 44,9 \\
$\quad 60-69$ & & 45 & 38,2 \\
$\quad 70-79$ & 117 & 20 & 16,9 \\
$\quad \geq 80$ & & 48 & 41,0 \\
Situação Conjugal & 117 & 69 & 59,0 \\
$\quad$ Com companheiro (a) & & & 77,8 \\
$\quad$ Sem companheiro (a) & & 26 & 22,2 \\
$\quad$ Cor da pele & 114 & & 3,5 \\
$\quad$ Negro & & 4 & 96,5 \\
$\quad$ Não Negro & & 110 & \\
$\quad>8$ anos & & & 12,1 \\
$\quad$ < anos & & & 87,9 \\
$\quad$ Nunca estudou & 116 & &
\end{tabular}


Nível de atividade física

Suficientemente ativo

Insuficientemente ativo

Busca de serviços de saúde

$\geq 2$ vezes/ano

1 vez/ano

Nenhuma

\section{Colesterol total}

Normal

Alterado

Colesterol LDL

Normal

Alterado

IMC

Eutrófico

Baixo Peso

Sobrepeso

Comorbidades

Ausente

Presente

Medicamento para SM

Sim

Não

Interação Medicamentosa

Sim

Não

Quantidade de critérios da SM

3 critérios alterados

4 critérios alterados

5 critérios alterados

Consumo de álcool

Não

Sim

Uso de tabaco

Não

Sim
118

\section{3}

44,9

65

55,1

112

$96 \quad 85,8$

$8 \quad 7,1$

$8 \quad 7,1$

118

$51 \quad 43,2$

$67 \quad 56,8$

108

$54 \quad 50,0$

$54 \quad 50,0$

113

$34 \quad 30,1$

54,4

$74 \quad 65,5$

118

$1 \quad 0,8$

$117 \quad 99,2$

118

$56 \quad 47.5$

$62 \quad 52,5$

118

$32 \quad 27,1$

$86 \quad 72,9$

117

$60 \quad 51,3$

$41 \quad 35.0$

$16 \quad 13,7$

117

$100 \quad 85,5$

$17 \quad 14,5$

109

$103 \quad 94,5$

$6 \quad 5,5$

Fonte: Autores.

A Tabela 2 mostra os valores médios e desvio padrão dos exames laboratoriais e parâmetros clínicos que compõem os critérios de diagnóstico da SM. Ao analisar os resultados, percebe-se que o valor do triglicerídeos $(185,33 \mathrm{mg} / \mathrm{dl})$ encontrase superior ao valor de referência estabelecido pela I-DBSM (acima de $150 \mathrm{mg} / \mathrm{dl}$ ). Seguindo a mesma diretriz, ao avaliar o valor encontrado para a média da glicemia $(124,03 \mathrm{mg} / \mathrm{dl})$ e considerando que os pacientes estavam em jejum, nota-se que a média enquadra a amostra num perfil de hiperglicemia ou intolerância à glicose (glicemia em jejum $\geq 110 \mathrm{mg} / \mathrm{dL}$ ). A média em relação a circunferência abdominal $(102,43 \mathrm{~cm})$ demonstra ser superior ao estabelecido para as mulheres $(88 \mathrm{~cm}$ para mulheres e $102 \mathrm{~cm}$ para homens). 
Tabela 2 - Média dos exames laboratoriais e parâmetros clínicos de idosos com síndrome metabólica. Aiquara-BA, Brasil, 2019.

\begin{tabular}{lccc}
\hline Critérios SM & N & Média (DP) & Min - Max \\
\hline PAS* (mmHg) & 115 & $157,30( \pm 22,8)$ & $105-219$ \\
PAD $^{* *}(\mathrm{mmHg})$ & 115 & $88,28( \pm 12,3)$ & $64-120$ \\
Glicemia jejum (mg/dL) & 118 & $124,03( \pm 47,3)$ & $76-331$ \\
Colesterol HDL & 112 & $44,76( \pm 17,9)$ & $22-204$ \\
Triglicerídeos & 117 & $185,33( \pm 108,2)$ & $56-853$ \\
Circunferência Abdominal $(\mathrm{cm})$ & 113 & $102,43( \pm 10,9)$ & $77-133$ \\
\hline
\end{tabular}

** PAS: Pressão arterial sistólica; ** PAD: Pressão Arterial Diastólica, ** SM: Síndrome Metabólica. Fonte: Autores.

No que se refere ao perfil farmacoterapêutico, foram encontrados 59 diferentes tipos de medicamentos utilizados pelos idosos da pesquisa, sendo esses apresentados na Tabela 3, segundo a classificação ATC (OMS, 2009). Nota-se que fármacos para o aparelho cardiovascular $(28,8 \%)$ e aqueles utilizados para o tratamento do aparelho digestivo e metabolismo $(20,3 \%)$ representam maior percentual do total dos medicamentos utilizados pelos idosos estudados.

Tabela 3 - Classificação dos medicamentos utilizados por idosos com síndrome metabólica, conforme a Anatomical Therapeutic Chemical (ATC), nível 1.Aiquara-BA, Brasil, 2015.

\begin{tabular}{lc}
\hline Classificação segundo a ATC & FR $(\boldsymbol{\%})$ \\
\hline A - aparelho digestivo e metabolismo & 20,3 \\
B - sangue e órgãos hematopoéticos & 6,8 \\
C - aparelho cardiovascular & 28,8 \\
D - medicamentos dermatológicos & 1,7 \\
G - aparelho geniturinário e hormônios sexuais & 1,7 \\
J - aparelho respiratório & 3,4 \\
M - sistema músculo esquelético & 1,7 \\
N - sistema nervoso & 6,8 \\
P - antiparasitário, inseticida e repelente & 1,7 \\
S- órgãos sensitivos & 1,7 \\
R- aparelho respiratório & 10,2 \\
V - vários & 15,2 \\
\hline
\end{tabular}

FR: frequência relativa. Fonte: Autores.

A análise das interações medicamentosas de acordo com o software Medscape ${ }^{\circledR}$, obteve-se um total de 145 IMP, sendo a média equivalente a 4,5 IMP por idoso. Quanto à classificação da gravidade destas IMPs, 76,6\% apresentavam 'necessidade de monitoramento', 13,2\% foram classificadas como 'menor' e 10,2\% como 'graves'.

Em relação ao mecanismo de interação, 45,3\% foi do tipo farmacodinâmico, 28,9\% desconhecido seguido por 25,8\% farmacocinético. Os fármacos que apresentaram maior frequência quando comparados ao total de IMP foram o ácido acetilsalicílico (AAS) (31,6\%), digoxina (22,3\%), losartana (20,5\%), furosemida (20,2\%), espironolactona (12,2\%), carvedilol $(12,2 \%)$ e metformina (10,6\%). As demais IMP encontradas nesta população estão descritas na Tabela 4, sendo mais observadas as interações entre fármacos que são utilizados de forma contínua. 
Tabela 4 - Caracterização das interações medicamentosas potencias em idosos com síndrome metabólica. Aiquara-BA, Brasil, 2015 .

\begin{tabular}{|c|c|c|c|}
\hline Fármacos envolvidos nas IMP & Mecanismo & Efeitos adversos & $\%$ \\
\hline \multicolumn{4}{|c|}{ Menor } \\
\hline Metformina $\mathrm{x}$ furosemida & Não especificado & Hipoglicemia & 5,5 \\
\hline Ácido ascórbico x AAS & Farmacocinético & Redução da depuração renal & 2,0 \\
\hline Cetaconazol x losartana & Farmacocinético & Hipertensão & 1,3 \\
\hline Sulfametazol x trimetropina & Farmacocinético & Taquicardia & 1,3 \\
\hline Salmeterol $\mathrm{x}$ furosemida & Farmacodinâmico & Redução do potássio sérico & 1,3 \\
\hline AAS $x$ alendronato & Farmacodinâmico & Risco ulcera no TGI & 0,6 \\
\hline Diazepan $\mathrm{x}$ acetominofeno & Farmacocinético & Hepatotoxicidade & 0,6 \\
\hline Hidroclorotiazida $\mathrm{x}$ glibenclamida & Farmacodinâmico & - & 0,6 \\
\hline \multicolumn{4}{|c|}{ Necessidade de monitoramento } \\
\hline AAS x losartana & Farmacodinâmico & $\begin{array}{l}\text { Aumento do potássio sérico; } \\
\text { hipertensão }\end{array}$ & 8,2 \\
\hline AAS $x$ furosemida & Farmacodinâmico & & 6,2 \\
\hline Digoxina $\mathrm{x}$ espirolactona & Farmacocinético & Hipotensão & 6,2 \\
\hline Digoxina x carvedilol & Farmacodinâmico & Bradicardia,hipotensão & 5,5 \\
\hline AAS $x$ espirolactona & Não especificado & Hipercalemia & 4,1 \\
\hline Nifedipino $\mathrm{x}$ metformina & Farmacodinâmico & Hipoglicemia & 2,7 \\
\hline Carvedilol x AAS & Farmacodinâmico & Hipercalemia & 2,7 \\
\hline Carvedilol $\mathrm{x}$ amiodarona & Farmacodinâmico & Hipotensão, bradicardia & 2,7 \\
\hline Digoxina $x$ AAS & Não especificado & Hipercalemia & 2,7 \\
\hline Digoxina $\mathrm{x}$ furosemida & Não especificado & Hipocalemia, deterioração renal & 3,4 \\
\hline Glibenclamida x AAS & Farmacodinâmico & Hipoglicemia & 2,7 \\
\hline Digoxina $\mathrm{x}$ losartana & Não especificado & Hipercalemia & 2,0 \\
\hline Losartana $\mathrm{x}$ furosemida & Não especificado & Hipocalemia & 1,3 \\
\hline Atenolol x valsartana & Farmacodinâmico & $\begin{array}{l}\text { Comprometimento fetal durante } \\
\text { a gravidez; Hipercalemia }\end{array}$ & 1,3 \\
\hline Hidroclorotiazida x losartana & Não especificado & Hipocalemia & 1,3 \\
\hline Carvedilol x losartana & Farmacodinâmico & $\begin{array}{l}\text { Comprometimento fetal durante } \\
\text { a gravidez, hipotensão }\end{array}$ & 1,3 \\
\hline Espirolactona $\mathrm{x}$ losartana & Não especificado & Hipercalemia & 1,3 \\
\hline Atenolol x losartana & Farmacodinâmico & $\begin{array}{l}\text { Comprometimento fetal durante } \\
\text { a gravidez, hipotensão }\end{array}$ & 1,3 \\
\hline Enalapril $\mathrm{x}$ furosemida & Farmacodinâmico & Hipotensão, insuficiência renal & 1,3 \\
\hline Enalapril x glibenclamida & Farmacodinâmico & $\begin{array}{l}\text { Hipotensão aguda, insuficiência } \\
\text { renal }\end{array}$ & 1,3 \\
\hline Losartana $\mathrm{x}$ amiodarona & Farmacocinético & Hipotensão & 1,3 \\
\hline Losartana $\mathrm{x}$ metoprolol & Farmacodinâmico & $\begin{array}{l}\text { Comprometimento fetal durante } \\
\text { a gravidez }\end{array}$ & 1,3 \\
\hline Propanolol x hidroclorotiazida & Não especificado & Hipocalemia & 1,3 \\
\hline Atorvastatina $\mathrm{x}$ espirolactona & Farmacocinético & $\begin{array}{l}\text { Aumento dos efeitos da } \\
\text { atorvastatina }\end{array}$ & 0,6 \\
\hline AAS x clopidogrel & Farmacodinâmico & Aumento da toxicidade & 0,6 \\
\hline AAS x Doxazosina & Farmacodinâmico & $\begin{array}{l}\begin{array}{l}\text { Redução dos efeitos da } \\
\text { doxazosina }\end{array}\end{array}$ & 0,6 \\
\hline AAS $x$ fluoxetina & Farmacodinâmico & $\begin{array}{l}\text { Aumento do risco de } \\
\text { sangramento do TGI }\end{array}$ & 0,6 \\
\hline AAS $x$ salmeterol & Não especificado & Hipocalemia & 0,6 \\
\hline Atenolol $\mathrm{x}$ nifedipino & Não especificado & Hipotensão & 0,6 \\
\hline Atenolol $\mathrm{x}$ hidroclorotiazida & Não especificado & Hipocalemia & 0,6 \\
\hline Atorvastatina $\mathrm{x}$ digoxina & Farmacocinético & Hipertensão e toxicidade & 0,6 \\
\hline Dexclorfeniramina x salmeterol & Não especificado & Redução da sedação & 0,6 \\
\hline Omeprazol x losartana & Farmacocinético & Hipertensão & 0,6 \\
\hline Omeprazol x sulfato ferroso & Farmacocinético & Aumento do $\mathrm{pH}$ gástrico & 0,6 \\
\hline Metformina $\mathrm{x}$ insulina aspart & Farmacodinâmico & Sinergismo- hipoglicemia & 0,6 \\
\hline Anlodipino x metformina & Farmacodinâmico & Hipoglicemia & 0,6 \\
\hline Atenolol $\mathrm{x}$ anlodipino & Não especificado & Hipotensão & 0,6 \\
\hline Atenolol $\mathrm{x}$ furosemida & Não especificado & Hipocalemia & 0,6 \\
\hline
\end{tabular}




\begin{tabular}{|c|c|c|c|}
\hline Enalapril x metformina & Não especificado & Aumento da toxicidade & 0,6 \\
\hline Esperolactona $\mathrm{x}$ furosemida & Não especificado & Hipocalemia & 0,6 \\
\hline Valsartana $\mathrm{x}$ cetaconazol & Farmacocinético & $\begin{array}{l}\text { Redução dos efeitos anti- } \\
\text { hipertensivos }\end{array}$ & 0,6 \\
\hline Captopril x hidroclorotiazida & Farmacodinâmico & Nefrotoxicidade & 0,6 \\
\hline Digoxina $\mathrm{x}$ metformina & Farmacocinético & Toxicicidade e acidose lática & 0,6 \\
\hline Digoxina $\mathrm{x}$ captopril & Não especificado & Toxicidade & 0,6 \\
\hline Sinvastatina $\mathrm{x}$ digoxina & Farmacocinético & Hipotensão & 0,6 \\
\hline \multicolumn{4}{|c|}{ Grave } \\
\hline Omeprazol x digoxina & Farmacocinético & Aumento do pH gástrico & 4,5 \\
\hline Amiodarona $\mathrm{x}$ digoxina & Farmacocinético & Hipotensão & 1,3 \\
\hline Eritromicina $\mathrm{x}$ sinvastatina: & Farmacocinético & $\begin{array}{l}\text { Aumento do risco de } \\
\text { rabdomiólise }\end{array}$ & 1,3 \\
\hline AnlodipinoxSinvastatina & Não especificado & Miopatia & 0,7 \\
\hline Nifedipino $\mathrm{x}$ sinvastatina & Farmacocinético & Miopatia & 0,6 \\
\hline Losartana $\mathrm{x}$ captopril & Farmacodinâmico & $\begin{array}{l}\text { Hipertensão, hipercalemia e } \\
\text { insuficiência renal }\end{array}$ & 0,6 \\
\hline Prometazina x Eritromicina: & Farmacocinético & Miopatia & 0,6 \\
\hline Amiodarona $\mathrm{x}$ sinvastatina & Farmacocinético & Miopatia & 0,6 \\
\hline
\end{tabular}

Fonte: Autores.

A associação das IMPs com as variáveis sociodemográficas, comportamentais e de condições de saúde dos idosos portadores de SM (Tabela 5), verificou-se que as IMP estão associadas significativamente com a busca de serviços de saúde ( $\mathrm{p}=0,04)$, sendo mais prevalente nos idosos que buscam o serviço 2 ou mais vezes por ano $(28,1 \%)$; com os critérios de diagnóstico para SM ( $\mathrm{p}=0,05)$, sendo mais prevalente nos idosos que apresentaram 5 critérios alterados $(50 \%)$; bem como o uso de tabaco $(\mathrm{p}=0,03)$, sendo maior nos que se declaram fumantes $(66,7 \%)$.

Tabela 5 - Prevalência de Interações Medicamentosas Potenciais em Idosos com síndrome metabólica e sua associação com as variáveis independentes do estudo.Aiquara-BA, Brasil, 2015.

\begin{tabular}{|c|c|c|c|}
\hline \multirow[t]{2}{*}{ Variáveis } & \multicolumn{2}{|c|}{ Prevalência de IMPs (\%) } & \multirow[t]{2}{*}{ p-valor* } \\
\hline & Ausente & Presente & \\
\hline Sexo & & & 0,47 \\
\hline Masculino & 70,6 & 29,4 & \\
\hline Feminino & 78,8 & 21,2 & \\
\hline Faixa etária & & & 0,45 \\
\hline $60-69$ & 75,5 & 24,5 & \\
\hline $70-79$ & 66,7 & 33,3 & \\
\hline$\geq 80$ & 80,0 & 20,0 & \\
\hline Situação Conjugal & & & 0,71 \\
\hline Com companheiro (a) & 70,8 & 29,2 & \\
\hline Sem companheiro (a) & 73,9 & 26,1 & \\
\hline Cor da pele & & & 0,66 \\
\hline Negro & 73,6 & 26,4 & \\
\hline Não Negro & 69,2 & 30,8 & \\
\hline Escolaridade (anos de estudo) & & & 0,48 \\
\hline$>8$ anos & 100,0 & 0,0 & \\
\hline$<8$ anos & 72,7 & 27,3 & \\
\hline Nunca estudou & 73,7 & 26,3 & \\
\hline Renda** & & & 0,07 \\
\hline$>1$ salário mínimo & 92,9 & 7,1 & \\
\hline$\leq 1$ salário mínimo & 69,6 & 30,4 & \\
\hline Nenhuma & 72,4 & 27,6 & \\
\hline Nível de atividade física (IPAQ) & & & 0,50 \\
\hline Suficientemente ativo & 69,8 & 30,2 & \\
\hline Insuficientemente ativo & 75,4 & 24,6 & \\
\hline
\end{tabular}




\begin{tabular}{|c|c|c|c|}
\hline Busca de serviços de saúde & & & 0,04 \\
\hline$\geq 2$ vezes/ano & 71,9 & 28,1 & \\
\hline $1 \mathrm{vez} / \mathrm{ano}$ & 75,0 & 25.0 & \\
\hline Nenhuma & 75,0 & 25,0 & \\
\hline Colesterol total & & & 0,73 \\
\hline Normal & 74,5 & 25,5 & \\
\hline Alterado & 71,6 & 28,4 & \\
\hline Colesterol LDL & & & 0,67 \\
\hline Normal & 70,4 & 29,6 & \\
\hline Alterado & 74,1 & 25,9 & \\
\hline IMC & & & 0,59 \\
\hline Eutrófico & 76.5 & 23,5 & \\
\hline Baixo Peso & 80,0 & 20,0 & \\
\hline Sobrepeso & 70,3 & 29,7 & \\
\hline Comorbidades & & & 0,54 \\
\hline Ausente & 100,0 & 0,0 & \\
\hline Presente & 72,6 & 27,4 & \\
\hline Quantidade de Critérios da SM & & & $\mathbf{0 , 0 5}$ \\
\hline 3 critérios alterados & 80,0 & 20,0 & \\
\hline 4 critérios alterados & 73,2 & 26,8 & \\
\hline 5 critérios alterados & 50,0 & 50,0 & \\
\hline Consumo de álcool & & & 0,84 \\
\hline Não & 73.0 & 27,0 & \\
\hline Sim & 70,6 & 29,4 & \\
\hline Uso de Tabaco & & & 0,03 \\
\hline Não & 73,8 & 26,2 & \\
\hline Sim & 33,3 & 66,7 & \\
\hline
\end{tabular}

*Teste qui-quadrado de Pearson para tabelas 2x2; teste qui-quadrado linear por linear para tabelas 3x2.

**Baseado no salário mínimo do ano de 2015 (R \$ 788,00)

Fonte: Autores.

\section{Discussão}

Os principais achados deste estudo apontam uma prevalência de IMP de 27,1\%, nos idosos estudados, o que pode ser preocupante ao se considerar as peculiaridades orgânicas, morfofisiológicas e funcionais, próprias do envelhecimento, somadas ao quadro patológico inerente a síndrome metabólica nesta população, o que sugere a necessidade de um monitoramento do uso de medicamentos mais efetivo. Houve maior prevalência nos idosos do sexo masculino, o que se diferencia dos estudos realizados com idosos que apresentavam SM (Tavares et al., 2018; Rocha, Melo \& Menezes, 2016). Isto reforça a necessidade do fortalecimento de políticas em saúde voltadas para o cuidado do homem e do idoso.

Ao comparar a prevalência de IMP aqui encontrada com outros estudos realizados com idosos, constata-se que maiores prevalências foram descritas por outros autores, como descrito por Tavares et al. (2018), ao avaliar idosos com SM no ambiente ambulatorial em Uberaba-MG, em que a prevalência de IMP foi de 75,3\%. Outro estudo, conduzido com idosos com SM, usuários de uma unidade básica de saúde em Vila Velha-ES, as IMP prevaleceram em 80\% dos indivíduos (Andrade et al., 2009). Essas divergências podem ser explicadas devido a possíveis diferenças socioeconômicas, perfil das populações estudadas, tamanho da amostra, métodos de diagnósticos da SM e também variações nos métodos de identificação das IMP.

Neste estudo a presença de IMP teve associação significativa com a busca de serviços de saúde $(\mathrm{p}=0,04)$, sendo mais prevalente nos idosos que buscam o serviço duas ou mais vezes por ano $(28,1 \%)$ e com os critérios de diagnóstico para SM $(\mathrm{p}=0,05)$, sendo mais prevalente nos idosos que apresentaram cinco componentes da síndrome alterados $(50 \%)$. Esses fatores podem estar inter-relacionados no sentido de que sendo maior o número de morbidades associadas a SM, acredita-se que seja maior também a necessidade de consultas médicas e intervenções assistenciais que demandam maior número de medicamentos prescritos, inclusive em regime de polifarmácia, para tratar cada componente alterado de forma isolada (Carvalho et al., 2012), o que consequentemente, aumenta o número de interações medicamentosas possíveis. 
Ademais, houve associação significativa com o uso de tabaco ( $\mathrm{p}=0,03)$, sendo maior nos que se declaram fumantes (66,7\%). Para Carvalho et al. (2013), esse fator aumenta a probabilidade de IMP devido a insuficiência dos sistemas excretores dos idosos, que faz com que sua permanência no organismo seja aumentada, interferindo na ação dos fármacos. Haja vista o uso de tabaco como um importante fator de risco para o agravamento das DCNTs faz-se necessário aumentar os esforços para a conscientização da cessação deste hábito por estes idosos. Soma-se a isso, a própria característica sociodemográfica da população estudada, onde a baixa escolaridade e renda insuficiente são fatores que podem corroborar para o agravamento do quadro clínico, aumento de comorbidades e necessidade de polimedicação. Esses achados diferenciam-se do estudo de Tavares et al. (2018) realizado entre idosos com SM, onde os principais fatores associados às IMPs foi a faixa etária de 75 anos ou mais, a presença de polifarmácia e a utilização de medicamentos potencialmente inapropriados.

Quanto à média de IMP por paciente $(4,5)$, o resultado encontrado revela ser superior, quando comparado ao estudo feito por Amaral e Perassolo (2012) que obtiveram uma média de 3,7 IMP em idosos hipertensos e diabéticos, atendidos no grupo Hiperdia, em um município do Rio Grande do Sul. Santos; Junior e Restini (2012) avaliaram as prescrições de hipertensos de Pradópolis-SP e identificaram 2,4 IM por paciente. Isso demonstra que, apesar da menor prevalência geral, os idosos investigados neste estudo apresentam alta propensão a efeitos colaterais e complicações associadas às interações medicamentosas.

Ao analisar o perfil das IMP, verificou-se que aquelas envolvendo o AAS apresentaram alta prevalência (31,6\%), e corrobora com o estudo feito por Garske (2016) que investigou as interações medicamentosas potenciais na farmacoterapia de idosos atendidos em farmácia básica do sul do Brasil, relatando alta prevalência de IMPs envolvendo esse fármaco. Contudo é importante salientar que há uma limitação no software utilizado, visto que ele não leva em consideração a dose do fármaco, o que faz das IMP envolvendo o AAS serem questionáveis, visto que ela é dependente da dose utilizada, que na clínica pode ser empregada tanto para finalidade antitrombótica quanto anti-inflamatória (Alves et al., 2019).

Outro fármaco que se destacou neste estudo por estar envolvido na maioria das interações foi a digoxina que, e é importante destacar que sua utilização deve ocorrer de forma monitorada, pois este fármaco apresenta estreito índice terapêutico, o que contribui para o alto risco de intoxicação, principalmente em idosos, devido às alterações farmacocinéticas que ocorrem com o envelhecimento e comprometem em especial a metabolização e eliminação.

No que se refere à classificação das IMPs de acordo com a gravidade, as que 'necessitam de monitoramento' foram as mais prevalentes, corroborando com o estudo de Alves et al. (2019) realizado com adultos e idosos em Juazeiro do Norte-CE, ao avaliar as IMP entre antihipertensivos e hipoglicemiantes orais. Gotardelo et al. (2014), verificou as IMPs em um estudo de base populacional com idosos de Timóteo-MG e obteve resultado semelhante. Cabe salientar que, para o paciente a presença de IMP que necessita de monitoramento pode resultar em deterioração no estado clínico, na necessidade de introdução de um tratamento adicional, hospitalização ou aumento do tempo de permanência hospitalar (Moura et al., 2016).

Neste sentido, as interações classificadas neste nível de gravidade, podem gerar diversas complicações que culminam na diminuição ou perda da eficiência de alguns anti-hipertensivos no manejo da hipertensão arterial sistêmica, importante componente da SM. A interação entre o AAS e a losartana observada neste estudo, pode gerar o comprometimento da ação anti-hipertensiva do antagonista de receptores de angiotensina, devido ao antagonismo farmacodinâmico, devido a inibição irreversível da enzima ciclooxigenase, causada pelo AAS, que tem como subprodutos as prostaglandinas, que é uma substância vasodilatadora. Apesar disso, acredita-se que o benefício relacionado à prevenção de problemas cardiovasculares seja superior ao risco dessa associação (Bibiana, Floriano e Borges, 2019). No tocante a classe farmacológica do losartana - antagonista do receptor de angiotensina II (ARA II), deve-se atentar ainda às interações a nível de metabolismo enzimático, visto que esses fármacos são amplamente metabolizados pelas isoenzimas do citocromo P450, podendo, em alguns casos, terem sua eficácia reduzida (Cavalcante et al., 2020). 
Outras IMP envolvendo este fármaco foram prevalentes e chama a atenção para a cautela no uso de AINEs nestes pacientes, pois eles podem interferir de uma forma geral na perda de eficácia da ação de diuréticos, como a furosemida (Scrignoli, Teixeira \& Leal, 2016) redução da eficácia da excreção de sódio (Bibiana, Floriano \& Borges, 2019), além de proporcionar o aumento do potássio sérico, podendo acarretar perda da eficácia do diurético, possível nefrotoxicidade e hipercalemia (Scrignoli, Teixeira \& Leal, 2016) fatos observados durante a associação do AAS com a espironolactona.

Aponta-se ainda a relevância do monitoramento da associação entre digoxina e espironolactona, uma vez que a digoxina é considerada um fármaco de alto risco na prática clínica, visto que ele é comumente associado a IMP. O efeito hipotensivo observado por esta associação, pode ser justificado pelo aumento das concentrações séricas da digoxina em cerca de $25 \%$ decorrente da sua inibição da excreção renal e, a toxicidade pelo seu estreito índice terapêutico, que compromete a segurança do paciente (Mibielli at al., 2014). Não obstante, destacam-se ainda os efeitos colaterais resultantes da associação entre a digoxina e o carvedilol, devido ao aumento dos níveis séricos da digoxina que resulta em hipotensão e bradicardia.

Ao avaliar as IMPs de gravidade 'menor' observadas neste estudo, destaca-se a interação entre metformina e furosemida, que de acordo com a literatura é considerada desejável quando há um quadro de diabetes, visto que, ocorre um efeito aditivo que é responsável pela redução da glicemia. Contudo, é necessário um acompanhamento constante no que concerne aos índices glicêmicos bem como ao aparecimento de efeitos colaterais (Santos; Junior \& Restini, 2012).

Ademais, o presente estudo corrobora com o realizado por Tavares et al. (2018) que verificou que as IMP de gravidade maior em idosos com SM foram as de menor prevalência. Via de regra, este tipo de IMP expõe os pacientes a efeitos que são potencialmente ameaçadores à vida ou são capazes de causar danos permanentes, devendo ser evitadas, sempre que possível (Moura et al., 2016). Destacou-se neste estudo a interação entre o omeprazol e a digoxina, que, de acordo com a literatura, o aumento do pH resultante desta associação reduz a hidrólise da digoxina, aumentando a sua absorção e elevando os níveis séricos deste fármaco, com risco de intoxicação cardíaca devido à estreita janela terapêutica (Mibielli et al, 2014).

Outra IMP de gravidade maior envolvendo este fármaco que chama a atenção nesta população é a existente na sua associação com a amiodarona, gerando um quadro de hipotensão devido ao aumento da concentração sérica da digoxina. O mecanismo farmacocinético que justifica esta IMP se dá pela inibição de enzimas do citocromo P450 pela amiodarona, reduzindo o clearance da digoxina e consequentemente aumentando sua concentração (Carvalho et al., 2013).

O presente estudo apresenta como limitações, a ausência de avaliação clínica de reações adversas relacionadas com as IMP, visto que os pacientes não foram acompanhados clinicamente após a coleta de dados, além da impossibilidade de detectar o grau de causalidade entre as associações encontradas, dado o delineamento transversal do mesmo; ressalta-se também que a ausência de algumas informações referentes à dose dos medicamentos no software utilizado para testar as IMP impossibilitou o conhecimento mais detalhado do mecanismo de algumas interações. Entretanto podemos destacar como pontos fortes a premissa de que os resultados aqui encontrados são úteis na identificação de IMP, no processo de prescrição de medicamentos bem como na elaboração de procedimentos e protocolos que minimizem e evitem inadequações de prescrições, levando-se em consideração as peculiaridades da faixa etária estudada. Dessa forma, os profissionais de saúde devem estar atentos a farmacoterapia utilizada pelos pacientes, de modo a propor intervenções diante do aparecimento de quaisquer eventos adversos relacionados ao uso de medicamentos na população idosa, sobretudo naqueles portadores da síndrome metabólica.

\section{Conclusão}

Conclui-se que os idosos com SM apresentaram uma prevalência de IMP considerável, estando associada a utilização de serviços de saúde, aos critérios de diagnósticos da SM e ao uso de tabaco. Observou-se que os fármacos envolvidos nas IMP são de utilização contínua pelos pacientes, o que evidencia a necessidade de monitoramento constante desses idosos por parte da equipe de saúde, principalmente pelo farmacêutico. 
Por conseguinte, nota-se a importância da identificação precoce das interações medicamentosas na farmacoterapia do paciente idoso, tendo em vista a perspectiva de implementar medidas terapêuticas que visem garantir a segurança do paciente e a efetividade durante o tratamento medicamentoso.

\section{Referências}

Alves, N. R., Menezes, P. D. L., Diniz, J. A., Souza, F. A. F., Carvalho, P. M. M., \& Tavares, S. M. Q. M. C (2019). Avaliação das interações medicamentosas entre antihipertensivos e hipoglicemiantes orais. Rev multidisciplinar e de psicologia, 13(44):374-392.

Amaral, D. M. D. D., \& Perassolo, M. S (2012). Possíveis interações medicamentosas entre os anti-hipertensivos e antidiabéticos em participantes do Grupo hiperdia de Parobé, RS (Uma análise teórica). Rev de Ciências Farmacêuticas Básica e Aplicada, 33(1):99-105.

Andrade, E. M., Cesana, E. S., Ferreira, N. G., Vitória, E. L., \& Andrade, T. U (2009). Identificação de problemas relacionados com medicamentos nos pacientes com síndrome metabólica atendidos em uma unidade básica de saúde do município de Vila Velha-ES. Rev de Ciências Farmacêuticas Básica e Aplicada, 28(3):291-299.

Barreto, M. S., Carreira, L., \& Marcon, S. S (2015). Envelhecimento populacional e doenças crônicas: Reflexões sobre os desafios para o Sistema de Saúde Pública. Rev Kairós Gerontologia, 18(1):325-339.

Bibiana, B. G., Floriano, S. R., \& Borges, M. S (2019). Avaliação das interações medicamentosas em prontuários de pacientes de uma unidade básica de saúde. Journal of applied pharmaceutical Sciences, 5(10):9-27.

Brasil (2005). I Diretriz brasileira de diagnóstico e tratamento da síndrome metabólica. Arq. bras. cardiol., 84(supl. 1):3-28.

Brasil (2012). Resolução $\mathrm{n}^{\circ}$. 466, de 12 de dezembro de 2012. Aprova diretrizes e normas regulamentadoras de pesquisas envolvendo seres humanos. Diário Oficial da União. 13 jun. 2013.

Carvalho, A. A. D., Gomes, L., Loureiro, A. L., \& Bezerra, A. J. C (2013). Controle do tabagismo em instituição de longa permanência para idosos: relato de experiência. Ciência \& Saúde Coletiva, 18(2):1119-1130.

Carvalho, M.F.C., Romano-Lieber, N.S., Bergsten-Mendes, G., Secoli, S. R., Ribeiro, E. Lebrão, M. L., \& Duart, Y.A. O (2012). Polifarmácia entre idosos do município de São Paulo-Estudo SABE. Rev Brasileira de Epidemiologia, 15(1):817-827.

Cavalcante, G. L., Silva, A. R. da, Silva, H. R. da, Lima, D. C. dos S., Barbosa, M. C. N. A., Benoliel Vasconcelos, A. C. A., Santos Filho, J. B. dos, Ribeiro, Y. C. F., Santana, L. S. O. S., Pereira Junior, J. L., \& Abreu, F. L. de. (2020). Investigation of possible drug interactions of angiotensin II receptor antagonists used in the treatment of hypertension. Research, Society and Development, 9(7), e195973682.

Coelho, J. M. F., Silva, A. S., Brito, E. X. dos S., Marques, E. M., Ponte, G. A., Gomes, Érica V. D., Lima, K. G., França, L. M. C., Lima, L. A. da S., Mêrces, M. C. das ., Sampaio, L. M. de A., Fernandes, B. de S., Pinheiro, I. M., Galvão, L. R., \& Galvão, C. R. (2021). Sedentarism and Metabolic Syndrome in users of a Family Health Unit in Salvador- BA. Research, Society and Development, 10(1), e56910112195.

Expert Panel on Detection, Evaluation and Treatment of High Blood Cholesterol in Adults (2001). Executive summary of the Third Report of the National Cholesterol Education Program (NCEP) Expert Panel on Detection, Evaluation and Treatment of High Cholesterol. JAMA, 285:2486-97.

Ferreira, A.S., \& Soler, O. (2020). Fortalecendo as estratégias de segurança do paciente: uma revisão integrativa sobre os processos de segurança de medicamentos. Research, Society and Development, 9(12), e129129564.

Folstein, M. F., Folstein, S. E., \& Mchugh, P. R (1975). "Mini-mental state" A practical method for grading the cognitive state of patients for the clinician. Journal of Psychiatric Research, 12(3):189-198.

Garske, C. C. D., Assis, M. P., Schneider, A. P. H., Machado, E. O., \& Morsch, L. M (2016). Interações medicamentosas potenciais na farmacoterapia de idosos atendidos em farmácia básica do sul do Brasil. Saúde (Santa Maria), 42(2):97-105.

Gotardelo, D. R., Fonseca, L. S., Masson, E. R., Lopes, L. N., Toledo, V. N., \& Faioli, M. A (2014). Prevalência e fatores associados a potenciais interações medicamentosas entre idosos em um estudo de base populacional. Rev Brasileira de Medicina de Família e Comunidade, 9(31):111-118.

Instituto Brasileiro de Geografia e Estatística - IBGE (2010). Sinopse do Censo Demográfico de 2010. Disponível em: <http://www.censo2010.ibge.gov.br/sinopse.> Acesso em 10 de out 2019.

Lima, C. R. O. C., Araújo, L. S., Lemaire, D. C., Rios, DL, Conceição, G. C. ; Brandão, N. A., \& Araújo, E. M. Q (2017). Associação entre níveis séricos de vitamina D e componentes da síndrome metabólica em pacientes atendidos no centro de estudos e atendimento dietoterápico da Universidade do Estado da Bahia. Ciências Médicas e Biológicas, 16(3):367-373.

Lipschitz, D. A (1994). Screening for nutritional status in the elderly. Primary Care, 21(1):55-67.

Malachias, M. V., Souza, W. K., Plavnik, F. L., et al. (2016). Sociedade Brasileira de Cardiologia. $7^{\text {a }}$ Diretriz brasileira de hipertensão arterial. Arq. Bras. Cardiol., 107(3 supl 3):1-83.

Mazo, G. Z., \& Benedetti, T. R. B (2010). Adaptação do questionário internacional de atividade física para idosos. Bras Cineantropom Desempenho Hum., 12(6):480-4.

Mibielli, P., Rozenfeld, S., Matos, G. C. D., \& Arcucio, F. A (2014). Interações medicamentosas potenciais entre idosos em uso dos anti-hipertensivos da Relação Nacional de Medicamentos Essenciais do Ministério da Saúde do Brasil. Cadernos de Saúde Pública, $30(1): 1947-1956$. 
Research, Society and Development, v. 10, n. 9, e29410918080, 2021

(CC BY 4.0) | ISSN 2525-3409 | DOI: http://dx.doi.org/10.33448/rsd-v10i9.18080

Moura, L. Z., Kusma, S. Z., Garcia, I. C., Loures, M. E. I. A. R., \& Souza, M. A. P (2016). Uso de beta-bloqueadores em pacientes maiores que 65 anos com insuficiência cardíaca sistólica. Rev Médica da UFPR, 3(3):142-147.

Multi-Drug Interaction Checker-Medscape Reference. Available on http://www. reference.medscape.com/drug-interactionchecker.

Petroski, E. L (2011). Antropometria: técnicas e padronizações. Editora Fountora, (5a ed.), 208p.

Ramos, L. R., Tavares, N. U. L., Bertoldi, A. D., Farias, M. R., Oliveira, M. A., Luiza, V. L., Pizzol, T. S. D., Arrais, P. S. D., \& Mengue, S. S (2016). Polifarmácia e polimorbidade em idosos no Brasil: um desafio em saúde pública. Rev Saude Publica, 50(supl 2):9s.

Rocha, F. L., Melo, R. L. P. D., \& Menezes, T. N. D (2016). Factors associate ed with metabolic syndrome am ong the elderly in the northeast of Brazil. Rev Brasileira de Geriatria e Gerontologia,19(6):978-986.

Santos, J. C., Junior, M. F., \& Restini, C. B. A (2012). Potenciais interações medicamentosas identificadas em prescrições a pacientes hipertensos. Rev da Sociedade Brasileira de clínica médica, 10(4):308-17.

Scrignoli, C. P., Teixeira, V. C. M. C., \& Leal, D. C. P (2016). Interações medicamentosas entre drogas mais prescritas em unidade de terapia intensiva adulta. Rev Brasileira de Farmácia Hospitalar e Serviços de Saúde, 7(2):26-30.

Tavares, D. S., Gomes, N. C., Rodriguês, L. R., \& Tavares, D. M. S (2018). Perfil de idosos com síndrome metabólica e fatores associados às possíveis interações medicamentosas. Rev Brasileira de Geriatria e Gerontologia, 21(2):164-175.

Veras, R. P. \& Oliveira, M (2018). Aging in Brazil: the building of a healthcare model. Rev Ciência \& Saúde Coletiva, 23(6):1929-36.

World Health Organization (2009). Collaborating Centre for Drug Statistics Methodology. Anatomical Therapeutic Chemical ATC/DDD Index 2009. Oslo: World Health Organization. 